\title{
Direct Current (DC) Bias Stress Characteristics of a Bottom-Gate Thin-Film Transistor with an Amorphous/ Microcrystalline Si Double Layer
}

\author{
Tae Hoon Jeong, Si Joon Kim, and Hyun Jae Kim ${ }^{+}$ \\ School of Electrical and Electronic Engineering, Yonsei University, Seoul 120-749, Korea
}

Received March 29, 2011; Revised June 16, 2011; Accepted June 28, 2011

\begin{abstract}
In this paper, the bottom-gate thin-film transistors (TFTs) were fabricated with an amorphous/microcrystalline Si double layer (DL) as an active layer and the variations of the electrical characteristics were investigated according to the DC bias stresses. Since the fabrication process of DL TFTs was identical to that of the conventional amorphous Si (a-Si) TFTs, it creates no additional manufacturing cost. Moreover, the amorphous/microcrystalline Si DL could possibly improve stability and mass production efficiency. Although the field effect mobility of the typical DL TFTs is similar to that of a-Si TFTs, the DL TFTs had a higher reliability with respect to the direct current (DC) bias stresses.
\end{abstract}

Keywords: Thin-film transistor, Double layer, Direct current (DC) bias stress, Reliability

\section{INTRODUCTION}

Amorphous $\mathrm{Si}$ (a-Si) thin-film transistors (TFTs) are widely used as switching devices for active-matrix liquid-crystal displays (AMLCD) due to their many advantages such as low cost and efficient mass production. Based on the success of a-Si, a-Si TFT has been intensively investigated for realizing a future activematrix light-emitting diode (AMOLED) display [1,2]. However, a-Si TFT is not ideally suited for applications, such as AMOLED backplanes, unlike the case of AMLCD. It is known to be mainly due to low mobility and TFT instability [3-6]. Some reporters have revealed that microcrystalline $\mathrm{Si}(\mu \mathrm{c}-\mathrm{Si}) \mathrm{TFTs}$ could be a good alternative [7]. The $\mu \mathrm{c}-\mathrm{Si}$ TFT is compatible with the a-Si manufacturing technology in terms of having a well-established manufacturing infrastructure. The performance of $\mu c-S i$ TFT is expected to have much higher field effect mobility [8]. Additionally, the stability of $\mu \mathrm{c}-\mathrm{Si}$ TFT is improved under the DC bias stress [9]. However, the $\mu \mathrm{c}$-Si has a relatively slow deposition rate,

${ }^{\dagger}$ Author to whom all correspondence should be addressed:

E-mail: hjk3@yonsei.ac.kr

Copyright $@ 2011$ KIEEME. All rights reserved. This is an open-access article distributed under the terms of the Creative Commons Attribution Non-Commercia
License (http:///creativecommons.org/licenses/by-nc/3.0) which permits unrestricted noncommercial use

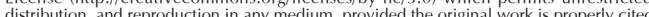

which is problematic for achieving efficient mass production. To address these problems, a bottom-gate TFT was proposed with an amorphous/ $\mu \mathrm{c}$-Si double layer (DL) as an active layer.

In this study, DL TFTs were fabricated and the variations of electrical characteristics were investigated according to direct current (DC) bias stresses.

\section{EXPERIMENTS}

The $\mu \mathrm{c}$-Si was deposited in a standard $13.56 \mathrm{MHz}$ capacitively coupled RF plasma-enhanced chemical vapor deposition (PECVD) reactor with a $\mathrm{SiF}_{4} / \mathrm{H}_{2} /$ inert gas mixture, which is known to induce microstructures into the nanocrystalline phases. A-Si was deposited with a $\mathrm{SiH}_{4} / \mathrm{H}_{2}$ gas mixture. A 4,000 $\AA$-thick Mo layer was deposited on glass by sputtering and then patterned for a gate electrode. Four layers of $\operatorname{SiN}_{\mathrm{x}}(4,000 \AA)$, a $\mathrm{SiF}_{4} / \mathrm{SiH}_{4}$-based DL (lower layer of $\mathrm{SiF}_{4}[1,000 \AA]$ / upper layer of $\left.\mathrm{SiH}_{4}[1,000 \AA]\right)$, and an $\mathrm{n}^{+} \mathrm{a}-\mathrm{Si}(500 \AA)$ layer were deposited on the gate electrode by PECVD. In case of a-Si TFT, a 2,000 $\AA$-thick a-Si layer was used instead of a DL layer. A 2,000 $\AA$-thick Mo layer was deposited on the $\mathrm{n}+\mathrm{a}$-Si and then patterned for a source/drain electrode. A 2,000 $\AA$-thick $\operatorname{SiN}_{\mathrm{x}}$ passivation layer was deposited on the source/drain electrode. Finally, the DL TFTs were fabri- 
(a)

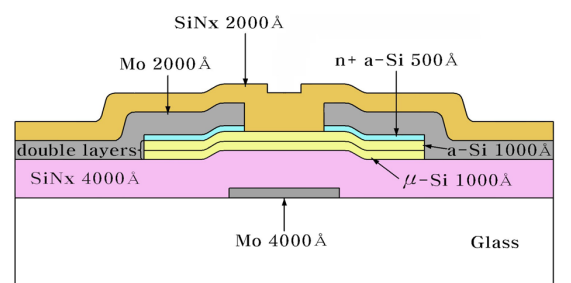

(b)

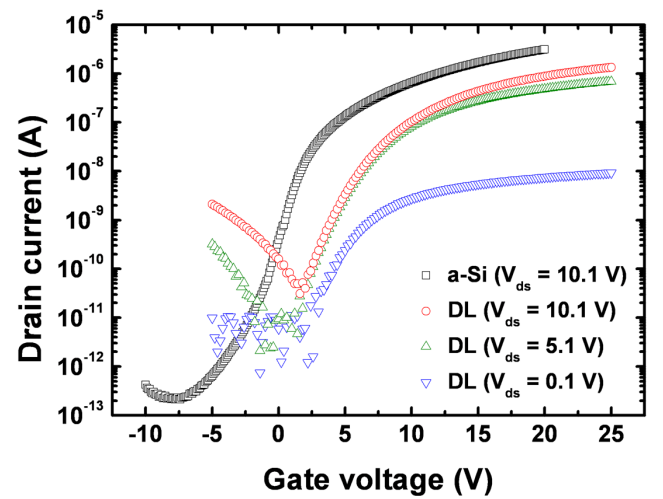

Fig. 1. (a) Schematic structure and (b) the typical transfer characteristics of a-Si thin-film transistor (TFT) and double layer (DL) TFT.

cated with $\mathrm{W} / \mathrm{L}=20 / 10 \mu \mathrm{m}$ as shown in Fig. 1(a).

\section{RESULTS AND DISCUSSION}

Figure 1(b) shows the typical transfer characteristics of a-Si TFT and DL TFT. The DL TFTs have field effect mobility within 0.5-0.7 $\mathrm{cm}^{2} / \mathrm{Vs}$ (measured at $\mathrm{V}_{\mathrm{ds}}$ of $5.1 \mathrm{~V}$ ), threshold voltages $\left(\mathrm{V}_{\mathrm{th}}\right)$ within 4-6 V, and subthreshold swings (S.S) within 1.2-1.8 V/ decade. The electrical characteristics of the DL TFTs are comparable to those of the conventional a-Si TFTs, except for the offcurrent levels. The abnormality of the off-current level may be caused by several causes, for example, source and drain region conductivity and active layer's thickness.

To study the DL TFT degradation, various DC bias stresses were applied to the substrates. Specifically, a-Si TFTs were fabricated to compare the degradation differences to DL TFT. The fabrication process of a-Si TFT was identical to that of DL TFT, except that the active layer was replaced with a 2,000 $\AA$-thick $\mathrm{SiH}_{4}$-based a-Si layer deposited by PECVD. The degradation mechanisms of a-Si TFTs have already been reported [10-12]. Degradation occurs by charge trapping in the gate insulator and by the creation of dangling bond defects in the channel near the Si-insulator interfaces [10]. Due to these degradation mechanisms, the $V_{\text {th }}$ shift was observed in a-Si TFTs with almost all stress conditions, and the shifts were significantly dominant in the positive gate bias stress.

Figure 2 shows the $V_{\text {th }}$ shift and mobility variations of the DL TFT and a-Si TFT after positive gate bias stress $\left(\mathrm{V}_{\mathrm{gs}}=30 \mathrm{~V}, \mathrm{~V}_{\mathrm{ds}}=0 \mathrm{~V}\right)$ for 10, 100, 1,000, 3,000, 6,000, and 10,000 seconds.

The DL TFTs do not show the $V_{\text {th }}$ shift degradation phenomenon, while the a-Si TFT suffers a significant $V_{\text {th }}$ shift of approximately $2.4 \mathrm{~V}$. The results of the positive gate bias stress indicate that the degradation mechanism of the DL TFT was not similar to that of a-Si TFT. Although the charge trapping in the SiNx layer and the creation of dangling bond defects in the Si-insulator interfaces are primary factors in the a-Si TFT degradation, in our results, charge trapping in the insulator layer could not be considered dominant because the SiNx deposition conditions were not changed between DL TFT and a-Si TFT. After 10,000 seconds, (a)

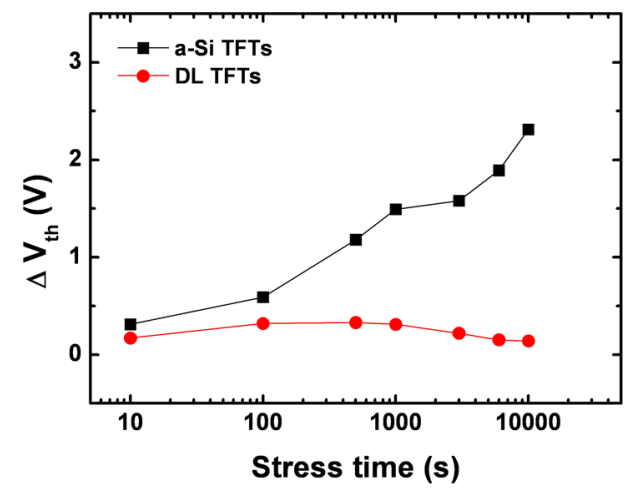

(b)

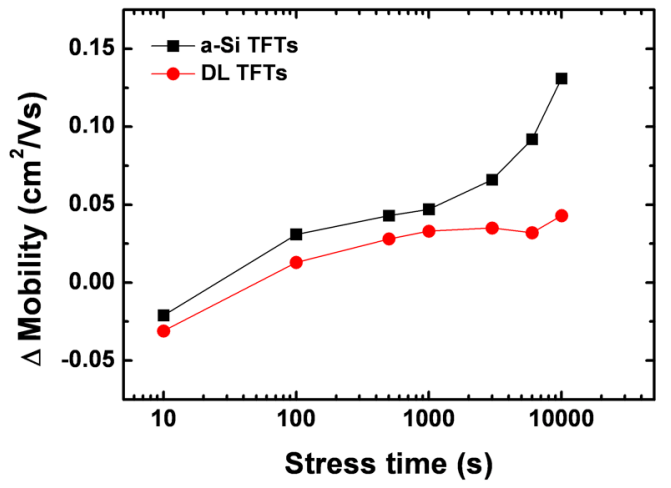

Fig. 2. (a) The $V_{\text {th }}$ shift and (b) the mobility variations of the double layer (DL) thin-film transistor (TFT) and a-Si TFT after positive gate bias stress $\left(\mathrm{V}_{\mathrm{gs}}=30 \mathrm{~V}, \mathrm{~V}_{\mathrm{ds}}=0 \mathrm{~V}\right)$ for $10,100,1,000,3,000,6,000$, and 10,000 seconds.

the a-Si TFT mobility decrease was measured as $0.13 \mathrm{~cm}^{2} / \mathrm{Vs}$, which was approximately two to three times larger than that of DL TFT. The mobility decrease could be attributed to defect generation, such as the breaking of Si-H bonds in the Si channel bulk rather than at the Si-insulator interface.

To study the defect generation in the Si channel in more detail, self-heating stress tests were conducted with the DL TFT and a-Si TFT within the linear region $\left(\mathrm{V}_{\mathrm{gs}}=20 \mathrm{~V}, \mathrm{~V}_{\mathrm{ds}}=10 \mathrm{~V}\right)$ and saturation region $\left(\mathrm{V}_{\mathrm{gs}}=20 \mathrm{~V}, \mathrm{~V}_{\mathrm{ds}}=20 \mathrm{~V}\right)$, respectively. It has been reported that the $V_{\text {th }}$ shift of a-Si TFT in the saturation region could be reduced with respect to the linear region [1]. As $\mathrm{V}_{\mathrm{ds}}$ increased, the charge injection amount at the Si-insulator interface was lowered, thus reducing the $V_{\text {th }}$ shift degradation in the a-Si TFT. Table 1 shows that the variations in transfer characteristics of the DL TFT and a-Si TFT were measured at $\mathrm{V}_{\mathrm{ds}}=5.1 \mathrm{~V}$ after self-heating stress with (a) $\mathrm{V}_{\mathrm{gs}}=20 \mathrm{~V}, \mathrm{~V}_{\mathrm{ds}}=10 \mathrm{~V}$, linear region stress and (b) $\mathrm{V}_{\mathrm{gs}}$ $=20 \mathrm{~V}, \mathrm{~V}_{\mathrm{ds}}=20 \mathrm{~V}$, saturation region stress for 10,000 seconds.

Under the same stress conditions, a-Si TFT had a large $\mathrm{V}_{\text {th }}$ shift and polycrystalline Si (poly-Si) TFT had joule heating mobility degradation [13]. In both bias stress conditions of DL TFT, a significant $\mathrm{V}_{\text {th }}$ shift was not observed. Additionally, the self-heating degraded DL TFT more in the linear region than in the saturation region, illustrating that the DL TFT has a degradation mechanism similar to a-Si TFT. However, the degradation of DL TFT is much less than that of a-Si TFT under these stresses conditions. On the other hand, in the self-heating operation of poly-Si TFT, degradation was characterized by i) a significant decrease in the on-current value and ii) a significant increase in the off-current value [13]. The research herein revealed that the DL TFT had not suffered the same degradation mechanisms encountered in previous research, suggesting the degradation mechanism was different than that of poly-Si TFT. To this end, the DL TFT was degraded with mechanisms similar to those of a-Si TFT under 


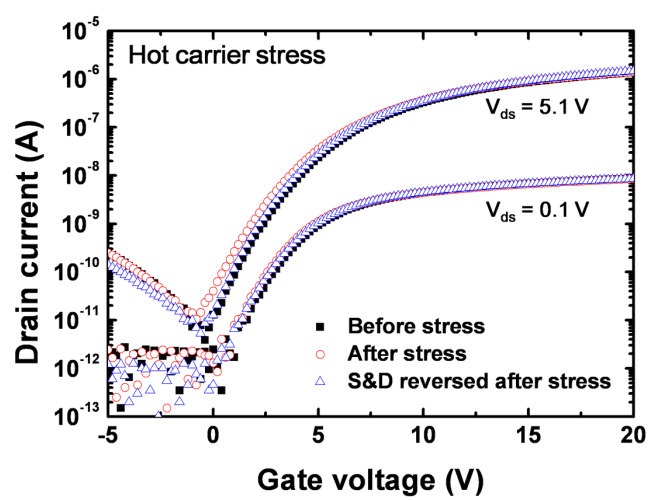

Fig. 3. The transfer characteristics of the double layer thin-film transistor measured at $\mathrm{V}_{\mathrm{ds}}=0.1 \mathrm{~V}$ and $\mathrm{V}_{\mathrm{ds}}=5.1 \mathrm{~V}$ before and after hot carrier stress $\left(\mathrm{V}_{\mathrm{gs}}=5 \mathrm{~V}, \mathrm{~V}_{\mathrm{ds}}=20 \mathrm{~V}\right.$, during 10,000 seconds $)$.

the self-heating stress condition. The self-heating degradation was known to be due to the breaking of Si-H bonding in the bulk $\mathrm{Si}$ channel rather than at the Si-insulator interface.

Figure 3 represents the transfer characteristics of the DL TFT measured at $\mathrm{V}_{\mathrm{ds}}=0.1 \mathrm{~V}$ and $\mathrm{V}_{\mathrm{ds}}=5.1 \mathrm{~V}$ before and after the hot carrier stress $\left(\mathrm{V}_{\mathrm{gs}}=5 \mathrm{~V}, \mathrm{~V}_{\mathrm{ds}}=20 \mathrm{~V}\right.$, during 10,000 seoncds). After the hot carrier stress, the off- and on-current indicated almost no degradation. The on-current characteristics of the DL TFT were nearly unchanged when measured at low drain voltage $\left(\mathrm{V}_{\mathrm{ds}}=0.1\right.$ $\mathrm{V})$, indicating the interface states were barely created during hot carrier stress condition.

When the source and drain contacts were reversed after the hot carrier stress, the off-current was slightly lower than the forward measurement. The reduction in current illustrates that the trap states near the drain region of DL TFT are locally created by impact ionization during the bias stress. Similar to DL TFT, the off- and on-current of a-Si TFT had almost no degradation at $\mathrm{V}_{\mathrm{ds}}$ $=0.1 \mathrm{~V}$ and $\mathrm{V}_{\mathrm{ds}}=5.1 \mathrm{~V}$ under hot carrier stress. The similarity under the hot carrier stress may be caused by the similar on-current levels of DL TFT and a-Si TFT, as degradation mainly depends on the applied source-drain voltages, hot carrier concentrations, and material characteristics. The degradation mechanism of poly-Si TFT was caused by the hot carrier stress, with a significant increase of off-current and decrease of on-current [14] Therefore, in terms of degradation mechanisms, DL TFT was unlike the poly-Si TFT.

\section{SUMMARY}

In summary, the DL TFTs, which were the same as the conventional a-Si TFTs process except that the active layer was replaced by amorphous/microcrystalline Si DL, were fabricated and the electrical characteristics under DC bias stresses were investigated. The DL TFT is directly applicable to the TFT fabrication process for commercial devices, ensuring efficient fabrication for mass production and is expected to improve the slow deposition rate of the active layer in $\mu \mathrm{c}$-Si TFT fabrication process, as the deposition rate of a-Si layer is much faster than $\mu \mathrm{c}$-Si. Because the degradation characteristics of DL TFT were unique, the degradation mechanism of DL TFT is explained unlike that of a-Si TFT or of poly-Si TFT. It is believed that the DL TFT degradation was mainly caused by the defect generation in the Si channel rather than charge trapping in the gate insulator or trap state creation in the region near the drain. It means that the DL TFT could
Table 1. Variations in transfer characteristics of the DL TFT and a-Si TFT measured at $\mathrm{V}_{\mathrm{ds}}=5.1 \mathrm{~V}$ after self-heating stress with (a) $\mathrm{V}_{\mathrm{gs}}=20 \mathrm{~V}$, $\mathrm{V}_{\mathrm{ds}}=10 \mathrm{~V}$, linear region stress and (b) $\mathrm{V}_{\mathrm{gs}}=20 \mathrm{~V}, \mathrm{~V}_{\mathrm{ds}}=20 \mathrm{~V}$, saturation region stress for 10,000 seconds.

\begin{tabular}{lcc}
\hline & DL TFT & a-Si TFT \\
\hline \hline Self Heating & $\Delta$ Mobility $=-0.076$ & $\Delta$ Mobility $=-0.32$ \\
(Linear) & $\Delta \mathrm{V}_{\text {th }}=0.32$ & $\Delta \mathrm{V}_{\text {th }}=1.45$ \\
& $\Delta \mathrm{S} . \mathrm{S}=0.24$ & $\Delta \mathrm{S} . \mathrm{S}=0.56$ \\
\hline \multirow{2}{*}{ Self Heating } & $\Delta$ Mobility $=-0.030$ & $\Delta$ Mobility $=-0.14$ \\
(Saturation) & $\Delta \mathrm{V}_{\text {th }}=0.06$ & $\Delta \mathrm{V}_{\text {th }}=0.7$ \\
& $\Delta$ S.S $=0.22$ & $\Delta \mathrm{S} . \mathrm{S}=0.43$ \\
\hline DL double layer TFT: thin-film transistor
\end{tabular}

achieve higher reliability than a-Si TFT or poly-Si TFT. Therefore, these desirable characteristics of DL TFTs might overcome the limitations of a-Si TFTs and $\mu \mathrm{c}-\mathrm{Si}$ TFTs.

\section{ACKNOWLEDGMENTS}

This work was supported by the National Research Foundation of Korea (NRF) through the National Research Laboratory Program grant funded by the Korean Ministry of Education, Science and Technology (MEST) [no. R0A-2007-000-10044-0(2007)].

\section{REFERENCES}

[1] T. Tsujimura, Jpn. J. Appl. Phys. 43, 5122 (2004) [DOI: 10.1143/ JJAP.43.5122].

[2] G. R. Chaji, S. J. Ashtiani, N. Safavian, and A. Nathan, Society for Information Display International Symposium, Digest of Technical Papers, p. 216 (2006).

[3] K. S. Girotra, Y. M. Choi, B. J. Kim, Y. R. Song, B. Choi, S. H. Yang, S. Kim, and S. Lim, J. Soc. Inf. Disp. 15, 113 (2007) [DOI: 10.1889/1.2709730].

[4] K. S. Girotra, J. H. Souk, K. Chung, S. K. Lim, S. Y. Kim, B. J. Kim, S. H. Yang, B. R. Choi, J. C. Goh, Y. R. Song, and Y. M. Choi, Society for Information Display International Symposium, Digest of Technical Papers, p. 1972 (2006).

[5] K. S. Jeong, S. J. Kwon, and E. S. Cho, J. of KIEEME 22, 1058 (2009).

[6] S. H. Kim, J. H. Hur, K. M. Kim, J. H. Koo, and J. Jang, J. Korean Phys. Soc. 48, S80 (2006).

[7] K. Y. Chan, J. Kirchhoff, A. Gordijn, D. Knipp, and H. Stiebig, Thin Solid Films 517, 6383 (2009) [DOI: 10.1016/ j.tsf.2009.02.101].

[8] C. H. Lee, A. Sazonov, and A. Nathan, Appl. Phys. Lett. 86, 222106 (2005) [DOI: 10.1063/1.1942641].

[9] P. Roca i Cabarrocas, R. Brenot, P. Bulkin, R. Vanderhaghen, B. Drevillon, and I. French, J. Appl. Phys. 86, 7079 (1999) [DOI: 10.1063/1.371795].

[10] H. Meiling and R. E. I. Schropp, Appl. Phys. Lett. 70, 2681 (1997) [DOI: 10.1063/1.118992].

[11] F. R. Libsch and J. Kanicki, Appl. Phys. Lett. 62, 1286 (1993) [DOI: 10.1063/1.108709].

[12] A. V. Gelatos and J. Kanicki, Appl. Phys. Lett. 57, 1197 (1990) [DOI: 10.1063/1.103484].

[13] S. Inoue, M. Kimura, and T. Shimoda, Jpn. J. Appl. Phys. 42, 1168 (2003) [DOI: 10.1143/JJAP.42.1168].

[14] N. D. Young, A. Gill, and M. J. Edwards, Semicond. Sci. Technol. 7, 1183 (1992) [DOI: 10.1088/0268-1242/7/9/007]. 